\title{
Personal fertility monitors for contraception
}

\author{
Thomas P. Bouchard MD, Stephen J. Genuis MD
}

I n 1990, Austrian chemist Carl Djerassi, who discovered the synthetic progestin used in the first birth control pill, expressed concern about existing contraceptive methods and predicted fertility awareness and family planning through biochemical measurement of endogenous hormones at home. ${ }^{1}$ Today, multiple fertility monitors are available that provide couples with accurate biochemical measurement of the fertile window to plan or avoid pregnancy, without potential side effects associated with other contraceptive methods. In this article, we review two new hormone-based personal fertility monitors that can be used for contraception.

Fertility monitoring involves biochemical identification of the fertile period and avoidance of intercourse, or use of other precautions, during this brief stage. The current understanding of the menstrual cycle is that an ovum in women of reproductive age is released once per cycle and is typically fertilizable for 12-24 hours, whereas sperm are typically viable for three to five days. In a series of studies by the World Health Organization in the $1970 \mathrm{~s}$, measurable changes in serum estradiol levels were found to signal the onset of potential fertility, and surges in luteinizing hormone levels were confirmed as the best predictor of impending ovulation. ${ }^{2}$ Subsequently, rising urine levels of estrone-3-glucuronide were found to correlate with rising serum estradiol levels and potential fertility, ${ }^{3}$ whereas surges in urine levels of luteinizing hormone accurately predicted impending ovulation. These findings showed that serum samples were no longer needed.

\section{How do fertility monitors work?}

The measurement of estrone-3-glucuronide and luteinizing hormone levels in urine to identify the fertile period was the premise in the development of two family-planning devices that first came to market about 10 years ago. The Persona monitor (Swiss Precision Diagnostics GmbH, Switzerland) was marketed for contraception; although currently available only in Europe, the device may be purchased in North America via the Internet.
The second device was marketed for assistance in achieving pregnancy; it is distributed as Clearblue Easy and Clearplan Easy in North America and Europe (Swiss Precision Diagnostics $\mathrm{GmbH}$ ) and as Clearview Primera in Japan (Mitsui Pharmaceuticals Inc., Japan). Using the same technology to indicate fertility, the Persona and Clearblue Easy fertility monitors use a urine-based test strip with antibodies to estrone-3-glucuronide and luteinizing hormone that detect elevated concentrations of these compounds.

Differences exist between the user instructions and sensitivity of these two fertility monitors. The Clearblue Easy monitor (Figure 1A) displays a "low," "high," or "peak" fertility reading, where "high" refers to an elevated urine estrone-3-glucuronide level and "peak" refers to the surge in urine luteinizing hormone level. Given that the Clearblue Easy monitor is designed as a tool to achieve pregnancy, its use to avoid conception should be considered "off label." As a contraceptive device, Persona (Figure 1B) displays a green light during the infertile phase and a red light during the fertile phase, during which it also displays a symbol to indicate the surge in luteinizing hormone and impending ovulation. The Persona monitor has a lower threshold for detection of estrone3-glucuronide and therefore indicates a slightly longer window of possible fertility before ovulation than the Clearblue Easy fertility monitor does. Accordingly, the Persona monitor may be more reliable for the minority of women who have short fertility phases.

\section{KEY POINTS}

- Hand-held devices that measure urine levels of estrone-3-glucuronide and luteinizing hormone can be used at home to determine the fertile period.

- Women may use these devices to prevent pregnancy by avoiding intercourse (or using other precautions) when increases in the hormone levels are detected.

- The Persona monitor has an effectiveness rate of $93.8 \%$ with correct use for avoiding pregnancy, and the Clearblue Easy monitor, in conjunction with observation of ovulatory mucous, has a correct-use effectiveness rate of $99 \%$.

- Although further study is required, the use of personal fertility monitors may be a useful option for family planning. 
Because the Clearblue Easy fertility monitor is readily available in North America and the Persona monitor has not yet been approved by the US Food and Drug Administration or Health Canada, the Clearblue Easy monitor has been more widely adopted in Canada and the United States.

\section{Who may benefit from using these devices?}

Based on our clinical experience and that of others, ${ }^{10}$ fertility monitors have been well received by women from a variety of backgrounds, but these devices appear particularly well suited to selected niches. For example, women with conditions such as a history of migraines, deep vein thrombosis, pulmonary embolus or stroke, may find fertility monitoring to be a welcome alternative to hormonal contraception. Women concerned about environmental issues and escalating levels of synthetic estrogens excreted into the water supply ${ }^{4}$ may also wish to avoid taking hormones by using these devices as an alternative to oral contraceptives. Furthermore, women disinclined toward
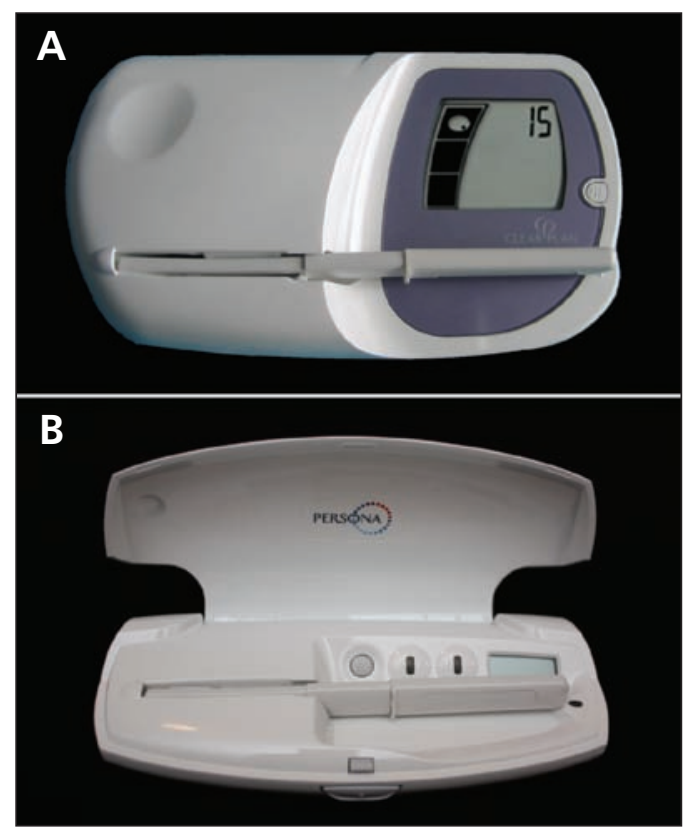

Figure 1: Effective use of the Clearblue Easy (A) and the Persona (B) fertility monitors requires three steps: 1. A button marked " $M$ " is pressed at the onset of menstruation. 2. Daily testing is performed by wetting a test stick with first morning urine and inserting the stick into the monitor. "High" and "peak" readings on the Clearblue Easy monitor, or a red light on the Persona monitor, indicate the fertile period. 3 . With the Clearblue Easy monitor, testing starts on day 6 and continues until the last "high" reading (usually 10-15 days per month); with the Persona monitor, testing starts on day 6 and continues for 16 days in the first cycle and for $\mathbf{8}$ days in each subsequent cycle. artificial methods of contraception for philosophical or religious reasons may find fertility monitoring to be consistent with their beliefs.

\section{What is the evidence so far?}

When used correctly, the Persona fertility monitor has an effectiveness rate of $93.8 \%$ for avoiding pregnancy. ${ }^{5}$ In addition, an Italian study showed that the fertile phase determined by the Persona monitor corresponded well with follicle growth and ovulation, as observed on ultrasound images and confirmed with serum luteinizing hormone and progesterone levels. ${ }^{6}$ In $94 \%$ of cycles, the first day of the potential fertile window (determined by ultrasonography) corresponded to the first instance of a red light displayed on the monitor; in $95.8 \%$ of cycles, ovulatory changes observed on ultrasound images occurred within the red-light period displayed on the monitor; and in $97.5 \%$ of cycles, the first day of infertility after ovulation observed on ultrasound images corresponded to the first post-ovulatory green light on the monitor. ${ }^{6}$

Similarly, a single-blinded prospective trial supported by the manufacturer correlated the readings on the Clearblue Easy fertility monitor with surges in serum luteinizing hormone levels and ovulation, as detected by transvaginal ultrasonography. ${ }^{7}$ The correlation showed that, $97 \%$ of the time, ovulation occurred within the two days of "peak" fertility plus one subsequent day of "high" fertility on the monitor, and no ovulations occurred before the monitor displayed a "peak" reading. ${ }^{7}$ In another study, urine levels of both estrone-3-glucuronide and luteinizing hormone measured using the Clearblue Easy monitor coincided well with serum measurements in identifying the potentially fertile phase. ${ }^{8}$ The exact effectiveness rates of using the Clearblue Easy monitor on its own for contraception have not yet been quantified. Therefore, the instructions for preventing pregnancy described in the caption to Figure 1 are based on clinical experience, not on evidence from randomized controlled trials.

Although studies showing positive and negative predictive values of the Clearblue Easy monitor for estrone-3-glucuronide measurement in relation to the possibility of pregnancy are lacking, a randomized controlled trial is currently underway to determine the daily probability of pregnancy, ${ }^{9}$ which will provide the sensitivity and specificity for the onset of fertility. Positive and negative predictive values for the onset of fertility with the Persona monitor have been calculated using the sensitivity and specificity values from the Italian study. ${ }^{6}$ The Persona monitor has a positive predictive value of $95.9 \%$ and a negative predictive value of 
$94.1 \%$. Table 1 summarizes the sensitivity and specificity of the two fertility monitors.

Although the predictive values for the onset of fertility using the Clearblue Easy fertility monitor have not yet been firmly established, as they have with the Persona monitor, a method of evaluating its effectiveness has been described in the literature. The "Marquette method" involves using the Clearblue Easy monitor in conjunction with observations of pre-ovulatory cervical mucus. An independent 12-month retrospective trial of this method involving 204 women revealed an effectiveness rate of $90.8 \%$ (95\% confidence interval [CI] 83\%-98\%) with typical use and $99 \%(95 \%$ CI $90 \%-100 \%)$ with correct use. ${ }^{10}$ A satisfaction questionnaire in this study showed good overall satisfaction with the method; the mean response was 3.0 on a fourpoint scale, where 1 was "not satisfied," 2 was "unsure," 3 was "satisfied" and 4 was "very satisfied." A study to evaluate discontinuation rates with the Marquette method showed that $1.5 \%$ stopped for health reasons, $9.1 \%$ stopped for personal reasons, $9.7 \%$ stopped to pursue pregnancy and $15.4 \%$ were lost to follow-up. ${ }^{11}$

In a more recent retrospective cohort study comparing family planning techniques, the Marquette method was associated with a pregnancy rate of $2.0 \%$ with correct use and $12 \%$ with typical use; it was shown to be more effective than observation of cervical mucus alone. ${ }^{12}$ Most of the participants in these trials, however, are white married couples in their late twenties; as such, the effectiveness rate with typical use of the device may not be applicable to all populations. Independent, prospective randomized controlled trials are underway to replicate these outcomes.

\section{What are the possible harms?}

Although safety and freedom from adverse effects are strong features of fertility monitors, they have limitations, as does any innovation (Table 2). Just as oral contraceptives require regular intake to be effective, fertility monitors require regular urine sampling on 10-15 days of a woman's cycle. If couples choose to have intercourse on "high" or "peak" days using the Clearblue Easy fertility monitor, or "red" days using the Persona monitor, alternate protection may be required to avoid unintended pregnancy.

The Clearblue Easy fertility monitor may be less reliable in women with short fertile phases. These women would benefit from the longer window defined by the Persona monitor, a doublecheck method as provided by the Marquette method or additional forms of protection at the onset of the fertile phase.
The financial outlay involves the cost of acquiring the monitor and the cost of purchasing monthly test strips. Fertility monitors are not yet covered on any Canadian formularies, but they may be eligible for coverage under private health insurance plans.

As with other nonbarrier methods of contraception, no protection against sexually transmitted infections is offered when using the monitors on their own.

\section{What can we expect in the future?}

Modern couples now have the opportunity to consider various options for safe and effective family planning to suit a plurality of backgrounds, beliefs and aspirations. As user-friendly devices that require little instruction, the ClearBlue Easy and Persona fertility monitors offer innovative approaches to contraception. Although extensive efficacy studies on these

Table 1: Sensitivity and specificity of the Clearblue Easy and Persona fertility monitors in identifying the fertile phase

\begin{tabular}{|c|c|c|c|c|}
\hline \multirow{2}{*}{$\begin{array}{l}\text { Event } \\
\text { predicted }\end{array}$} & \multicolumn{2}{|c|}{$\begin{array}{l}\text { Clearblue Easy } \\
\text { Fertility Monitor }\end{array}$} & \multicolumn{2}{|c|}{ Persona } \\
\hline & Sensitivity & Specificity & Sensitivity & Specificity \\
\hline $\begin{array}{l}\text { Onset of } \\
\text { fertility }\end{array}$ & $\begin{array}{l}\text { Results } \\
\text { pending }\end{array}$ & $\begin{array}{l}\text { Results } \\
\text { pending }\end{array}$ & $94 \%{ }^{6}$ & $96 \%{ }^{6}$ \\
\hline Ovulation & $97 \%^{7}$ & $100 \%^{7}$ & $95.8 \%{ }^{6}$ & $\begin{array}{l}\text { Not } \\
\text { calculated }^{6}\end{array}$ \\
\hline
\end{tabular}

Table 2: Pros and cons of using a personal fertility monitor for family planning

\begin{tabular}{|ll|}
\hline Pros & \multicolumn{1}{c|}{ Cons } \\
\hline - Easy for patient to use & - Cost of test sticks is ongoing \\
- Easy and efficient for physician & (\$15/month) and is not yet covered \\
to provide instruction & by government formularies \\
- Appears to provide reliable and & - Added instruction is required for \\
effective family planning* & women with inconsistent ovulation \\
- No known adverse effects & (e.g., post partum) \\
- Affordable for most patients & - No protection against sexually \\
- No environmental contamination & transmitted infections \\
(as is associated with hormonal & - Intercourse during the fertile phase \\
contraception) & indicated on the monitor may lead \\
- No risk of interactions with & to unintended pregnancy \\
medications (e.g., anti-epileptic & - For the minority of women who \\
medications) & have a short fertility phase, the \\
- May be ethically or morally & Persona fertility monitor may be \\
acceptable for patients from & more reliable than the Clearblue \\
certain religious or philosophical & Easy monitor in indicating possible \\
perspectives & fertility before ovulation; these \\
& women may need to use added \\
& precautions during intercourse \\
& - Further randomized controlled trials \\
& are needed to confirm effectiveness \\
\hline *Based on preliminary studies. & \\
\hline
\end{tabular}




\section{HYDROMORPH Contin'q12h}

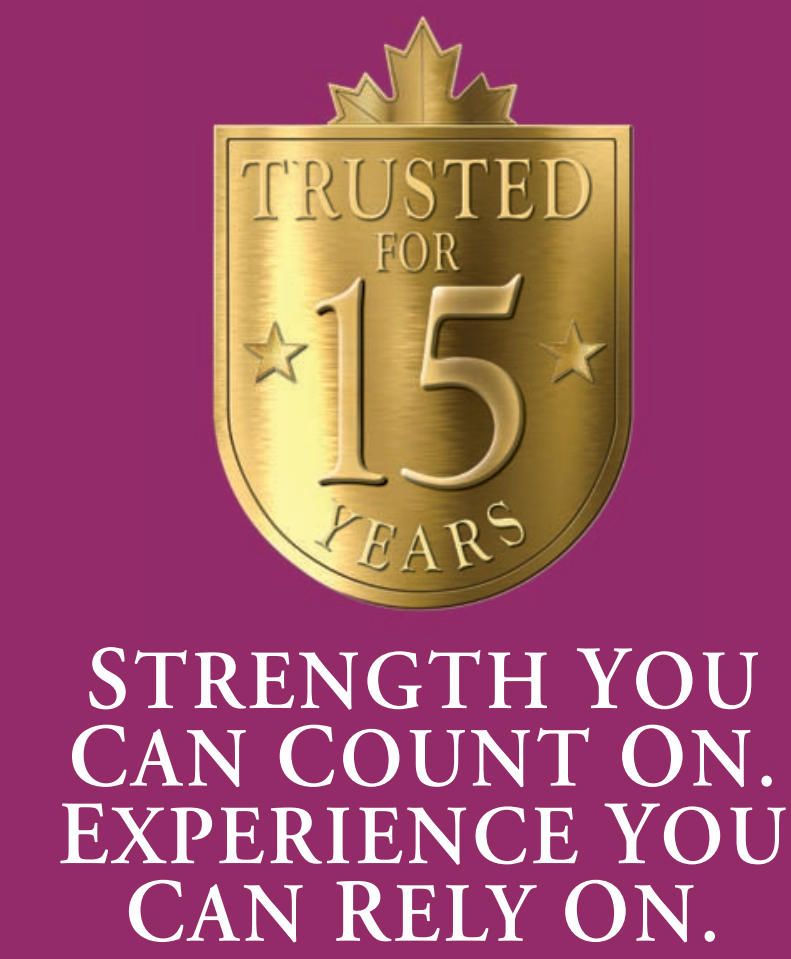

Hydromorph Contin ${ }^{\circledR}$ is indicated for the relief of severe chronic pain requiring the prolonged use of an oral opioid preparation.

Side effects are similar to other opioid analgesics. The most frequently observed are asthenic conditions, confusion, constipation, dizziness, lightheadedness, nausea, sedation, sweating and vomiting. Dosage limitations may be imposed by adverse effect. If they occur, please refer to prescribing information. Warning: Opioid analgesics should be prescribed and handled with a high degree of caution appropriate to the use of a drug with strong abuse potential. Patients should be cautioned not to consume alcohol while taking Hydromorph Contin ${ }^{\oplus}$, as it may increase the chance of experiencing dangerous side effects. Hydromorph Contin ${ }^{\circledR} 18 \mathrm{mg}$ capsules and higher are for use in opioid tolerant patients only. There is a potential for fatal respiratory depression in patients not previously exposed to similar equianalgesic doses of an opioid analgesic. Hydromorph Contin ${ }^{\circledR}$ capsules or capsule beads should not be chewed, crushed or dissolved since this can lead to rapid release and absorption of a potentially fatal dose of hydromorphone. Product monograph available on request.

Hydromorph Contin ${ }^{\circledR}$ capsule beads may be sprinkled on cold, soft food.

\section{'HYDROMORPH Contin'q12h \\ Controlled release hydromorphone capsules}

A trusted choice for severe chronic pain

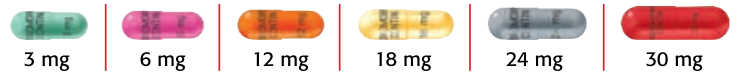

Initiation at $3 \mathrm{mg} \mathrm{q} 12 \mathrm{~h}$ or calculate the approximate daily oral hydromorphone dosage that should provide equivalent analgesia. See Product Monograph Table 1, Opioid Analgesics: Approximate Analgesic Equivalences.

devices are not yet available, the preliminary evidence of effectiveness is promising.

Given the risks associated with some forms of contraception and the emerging recognition of the potential impact of hormonal agents on the environment, fertility monitors may find a welcome place in the current array of contraceptive options. Although additional studies on efficacy are needed, these monitors appear to be well received, especially by women who are not able to, or choose not to, use other forms of family planning. Fertility monitors can be used without the need for extensive training, new skills or knowledge for prescribing clinicians. In addition, industry-produced materials are available on the use of these user-friendly devices, and minimal time or effort is required for instruction by health providers. For these reasons, fertility monitoring, as predicted by Dr. Djerassi, is expected to become increasingly popular as a method of reliable and safe family planning.

\section{References}

1. Djerassi C. Fertility awareness: Jet-age rhythm method? Science 1990;248:1061-2.

2. World Health Organization, Task Force on Methods for the Determination of the Fertile Period, Special Programme of Research, Development and Research Training in Human Reproduction. Temporal relationships between ovulation and defined changes in the concentration of plasma estradiol-17 beta, luteinizing hormone, follicle-stimulating hormone, and progesterone. I. Probit analysis. Am J Obstet Gynecol 1980;138:383-90.

3. Branch CM, Collins PO, Collins WP. Ovulation prediction: changes in the concentrations of urinary estrone-3-glucuronide, estradiol-17 beta-glucuronide and estriol-16 alpha-glucuronide during conceptional cycles. J Steroid Biochem 1982;16:345-7.

4. Ternes TA, Stumpf M, Mueller J, et al. Behavior and occurrence of estrogens in municipal sewage treatment plants - I. Investigations in Germany, Canada and Brazil. Sci Total Environ 1999;225:81-90.

5. Bonnar J, Flynn A, Freundl G, et al. Personal hormone monitoring for contraception. Br J Fam Plann 1999;24:128-34.

6. Guida M, Bramante S, Acunzo G, et al. Diagnosis of fertility with a personal hormonal evaluation test. Minerva Ginecol 2003; 55:167-73.

7. Behre HM, Kuhlage J, Gassner C, et al. Prediction of ovulation by urinary hormone measurements with the home use ClearPlan Fertility Monitor: comparison with transvaginal ultrasound scans and serum hormone measurements. Hum Reprod 2000;15:2478-82.

8. Tanabe K, Susumu N, Hand K, et al. Prediction of the potentially fertile period by urinary hormone measurements using a new home-use monitor: comparison with laboratory hormone analyses. Hum Reprod 2001;16:1619-24.

9. Pyper C, Bromhall L, Dummett S, et al. The Oxford Conception Study design and recruitment experience. Paediatr Perinat Epidemiol 2006;20(Suppl 1):51-9.

10. Fehring RJ, Schneider M, Barron ML. Efficacy of the Marquette Method of natural family planning. MCN Am J Matern Child Nurs 2008;33:348-54.

11. Fehring RJ, Schneider M, Raviele K, et al. Efficacy of cervical mucus observations plus electronic hormonal fertility monitoring as a method of natural family planning. J Obstet Gynecol Neonatal Nurs 2007;36:152-60.

12. Fehring RJ, Schneider M, Barron ML. Cohort comparison of two fertility awareness methods of family planning. J Reprod Med 2009;54:165-70.

Affiliations: From the Department of Family Medicine (Bouchard), University of Calgary Medical School, Calgary, Alta.; and the Department of Obstetrics and Gynecology (Genuis), University of Alberta, Edmonton, Alta.

Contributors: Both authors contributed substantially to the writing and revision of the manuscript for important intellectual content and approved the final version submitted for publication. 